This is the author's final manuscript of the presented paper. The publisher-formatted version may be available through the publisher's web site or your institution's library

\title{
High resolution wireless body area network with statistically synchronized sensor data for tracking pulse wave velocity
}

Kejia Li and Steve Warren

\section{How to cite this paper}

If you make reference to this version of the manuscript, use the following information:

Li, K., \& Warren, S. (2012). High resolution wireless body area network with statistically synchronized sensor data for tracking pulse wave velocity. Retrieved from http://krex.ksu.edu

\section{Published version information}

Citation: Li, K., \& Warren, S. (2012). High resolution wireless body area network with statistically synchronized sensor data for tracking pulse wave velocity. Engineering in Medicine and Biology Society (EMBC), Annual International Conference of the IEEE, 2012, 2080-2083. doi: $\underline{\text { 10.1109/EMBC.2012.6345853 }}$

Copyright: (02012 IEEE

Digital Object Identifier (DOI): doi: 10.1109/EMBC.2012.6345853

\section{Publisher's Link:}

http://ieeexplore.ieee.org/xpl/mostRecentlssue.jsp?punumber=6320834

This item was retrieved from the K-State Research Exchange (K-REx), the institutional repository of Kansas State University. K-REx is available at http://krex.ksu.edu 


\title{
High Resolution Wireless Body Area Network with Statistically Synchronized Sensor Data for Tracking Pulse Wave Velocity
}

\author{
Kejia Li, Student Member, IEEE and Steve Warren, Member, IEEE
}

\begin{abstract}
Wireless body area networks (WBANs) will take on more diverse forms in terms of their sensor combinations and communication protocols as their presence is extended to a greater number of monitoring scenarios. This paper presents an application layer protocol that solves issues caused by sensor nodes that must compete for high speed, real-time communication with the receiver. Such applications emphasize the delivery of large amounts of raw data from different sensor nodes in a time-synchronized manner, rather than channels that experience intermittent operation. An example of tracking pulse wave velocity (PWV) is introduced in this paper, where high-precision PWVs are estimated with the help of timeline recovery and feature extraction processes in MATLAB.
\end{abstract}

Keywords-photoplethysmogram, pulse wave velocity, statistical synchronization, wireless body area network

\section{INTRODUCTION}

$\mathrm{W}$ IRELESS body area networks have received significant attention in recent years due to their potential to enable patient-centered wellness monitoring, where the continuous acquisition of health indicators such as heart rate and activity level have garnered primary emphasis. Technical focus areas within this domain include sensor miniaturization [1], wearable sensors [2], ultra low-power circuits [3], communication protocols [4], and network topologies. General rules related to, e.g., low power and reliability, guide each WBAN development phase.

Since early 2008, the IEEE 802.15 Task Group 6 has addressed WBAN communication standards, which are likely to be based on the medium access control (MAC) layer of the current IEEE 802.15.4 standard [5]. Such a communication protocol should ensure high network capacity, energy efficiency, and adequate quality of service [6]. The protocol defines what data are transmitted and received, as well as when and how this occurs. To a large degree, these items determine the network performance, as they affect power consumption, data throughput, and packet loss rates. Data management techniques like adaptive duty cycles and message prioritization [4] can be considered if a monitoring scenario involves patient state/context changes and/or the coexistence of different types of sensor nodes.

Manuscript received March 15, 2012; accepted May 24, 2012. This work was supported in part by the National Science Foundation under grants BES-0093916, BES-0440183, and CNS-0551626. Opinions, findings, conclusions, or recommendations expressed in this material are those of the author(s) and do not necessarily reflect the views of the NSF.

Kejia Li and Steve Warren are with the Department of Electrical \& Computer Engineering, Kansas State University, Manhattan, KS 66506, USA (kejiali@ksu.edu; swarren@ksu.edu).
Diverse and creative application-layer schemes can be designed and evaluated for various WBAN scenarios. This paper presents a WBAN that emphasizes high-resolution raw data, real-time operation, and time synchronization of intrasensor data and inter-sensor waveforms. The WBAN requires a high-speed wireless connection that is always alive over the desired data acquisition period, as opposed to most WBAN applications, which allow periodic sleep modes. This type of WBAN transfers large amounts of raw data to a base station, where heavy signal processing is implemented. The demonstrative application in this paper is to precisely calculate pulse wave velocity based upon the small transit times of blood volume wavefronts between two sensor nodes at the wrist and fingertip, while larger scale tests are pending and the results are not verified against commercial medical equipment.

\section{BACKGROUND}

\section{A. Pulse Wave Velocity Estimation}

The authors recently presented a method to use two custom pulse oximeter sensors to estimate PWV from one hand [7]. The task was to extract several PWV values using waveform features from different stages of the cardiac cycle, given that time-varying intravascular pressures create timevarying PWVs. The pulse oximeter units used for this study provide high-fidelity photoplethysmograms (PPGs) sampled at $240 \mathrm{~Hz}$ per channel [8]. However, due to high PWVs and the relatively short distance between the measurement locations (the wrist and the corresponding fingertip), the number of valid PPG data points acquired during this small time window was limited.

\section{B. IEEE 802.15.4 MAC Layer}

The carrier sense multiple access with collision avoidance (CSMA/CA) method is adopted in the IEEE 802.15.4 MAC layer. The CSMA approach requires transmitting nodes to first listen to a channel and then transmit only if the channel is idle; the collision avoidance mechanism improves CSMA performance by dividing the communication channel equally among all nodes in the collision domain. Utilizing this method in a WBAN with only one channel ensures that each sensor node has an equal opportunity to upload data, and each successfully transmitted data segment can be synchronized with the lost data segments from the other nodes. Since ZigBee (the wireless standard utilized by the custom pulse oximeters) is based on IEEE 802.15.4, this approach is employed here. 


\section{METHODS}

\section{A. Study Description and Network Setup}

The experimental setup is similar to the earlier study [7] but improves on the approach by

- forming a star-topology WBAN with a single receiver and a single communication channel,

- doubling the sensor sampling frequency to $480 \mathrm{~Hz}$, theoretically doubling the resultant resolution, and

- using a curve fitting method over several cardiac cycles to build a more representative PPG segment, since PPG behavior statistically repeats over time.

The pulse oximeter sensor nodes transmit their own samples continuously (in real time), letting the CSMA/CA algorithm decide which sensor communicates to the receiver during a certain time slot. Data streams received from pulse oximeter sensor nodes are therefore fragmented, but these fragmented data segments are 'geared' together in a real-time manner. Note that since this approach aims to extract features from pulsatile high-resolution signals, the missed segments can be rebuilt afterwards.

\section{B. Issue 1: Lost and Wandering Frames}

Fig. 1 illustrates a star-topology ZigBee network with two sensor nodes (S1 and S2) and one receiver (Coordinator). Sensor-node frames are received by the Coordinator with equal probability. Each sensor has a unique MAC address, which can be identified by the Coordinator, so the frames sent by S1 and S2 are illustrated differently. Each frame is originally assigned a sequence \# to help address the issue of lost and wandering frames (see Fig. 1). Lost frames are unrecoverable because each sensor node only attempts to transmit a frame once to ensure real-time operation. Wandering frames can be rearranged by a computer, which receives frames from the Coordinator via a serial port.
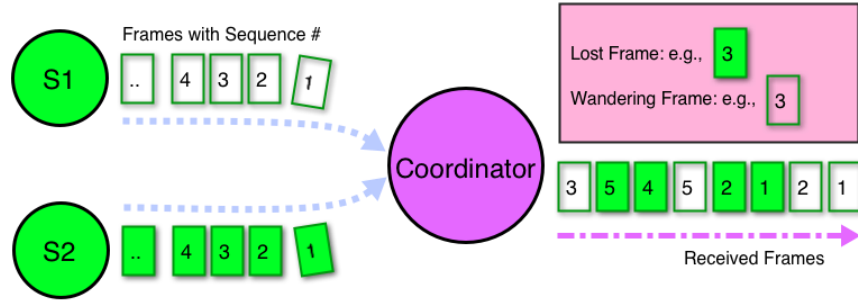

Fig. 1. An example star-topology network. Each frame sent by a sensor node carries a sequence \# to address the issue of lost and wandering frames.

\section{Issue 2: Timeline Distortion}

The second issue is caused by the asynchronization of the frame sending and receiving rates. For example, if S1 and S2 each competitively transmit 240 frames per second, the Coordinator does not necessarily receive a sum of 240 frames in one second. Experiments indicate that the number of received frames depends on the frame length.

To address this issue, the inner timers in the sensor nodes and the Coordinator are identical. Such timers trigger events like transmitting a frame or generating a ticket, as shown in Fig. 2. A Ticket Counter inside the Coordinator increases by 1 when the Ticket Generator produces a ticket and decreases by 1 when a received frame consumes a ticket. This Ticket Counter number is appended to each frame to assist the follow-on timeline restoration process. Continuing the thought, if the Ticket Counter value decreases by 10 in one second (the tickets are over-consumed), the Coordinator has received an extra 10 frames in one second, which means the timeline will be expanded given the assumption that 240 frames corresponds to one second. A correction factor of 0.96 , calculated from $240 /(240+10)$, should then be applied to the received-frame timeline.

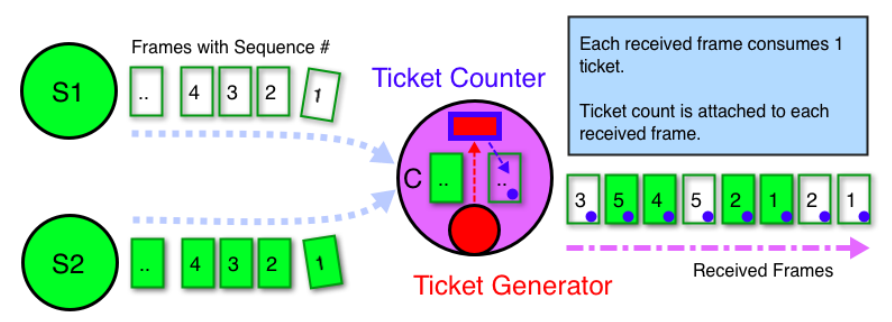

Fig. 2. Ticket production and consumption mechanism inside the Coordinator to address the timeline distortion issue.

\section{Frame Field Definition}

Frames are assembled on the application layer of the wireless communication protocol. Fig. 3 contains the frame field definitions for the sensor nodes (lower) and the Coordinator (upper). Each sensor frame carries data for two samples: their $\mathrm{AC}$ values and the corresponding $\mathrm{DC}$ value for the pair. The frame begins with the acknowledgement value returned from the previous transmission function and ends with a checksum. When the Coordinator receives a sensor frame, it adds an ID (mapped to the unique MAC address), a Ticket Count, and a new checksum.

\section{Coordinator (Receiver)}

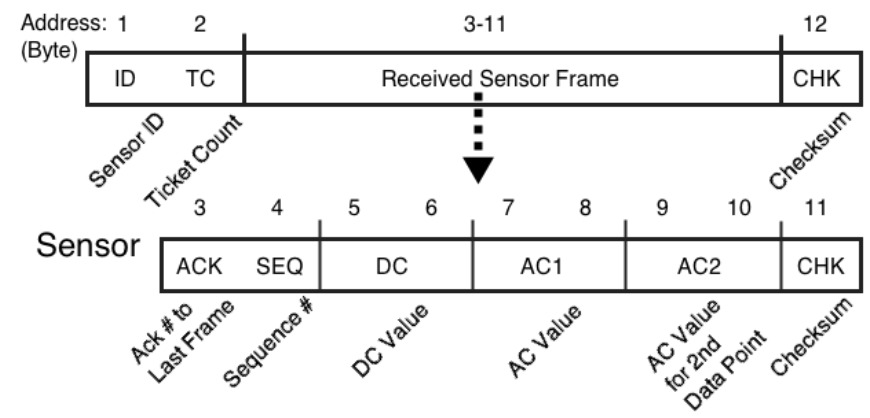

Fig. 3. Frame field definitions for the Coordinator and sensor nodes on the application layer of the custom communication protocol.

\section{E. Timeline Restoration in MATLAB}

The frames finally stream to a MATLAB interface on a computer, where all the "heavy" signal processing tasks are implemented. Task 1 is to arrange the frames from each sensor node in ascending order and fill the gaps with 'placeholder' frames - the positions that correspond to lost frames. Since a sequence \# is defined to be one byte long 
(see Fig. 3), the frame search window is assumed to be within $[-127,128]$.

Fig. 4 shows the frame sequence \#s in the received order (blue crosses) and the time-aligned sequence \#s (red dots) for one sensor. The original sequence illustrates that frame wandering occurs frequently. The number of placeholder frames (assigned with sequence \# = -1) between data segments is the length of data segments from other sensor nodes in the original sequence, and it will be equal to the actual number of lost frames in the recovered sequence if the frame search method did not miss any wandering frame.

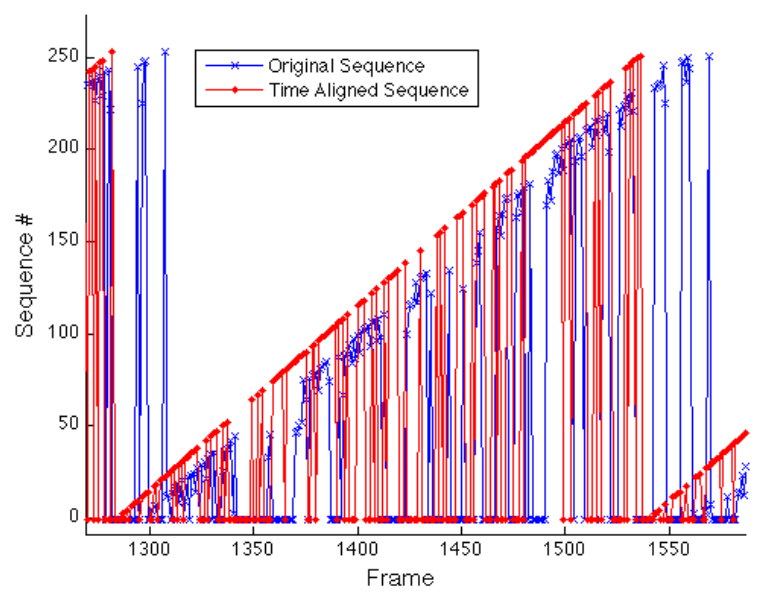

Fig. 4. Original data stream with sequence \#s in the received order (blue crosses) and the recovered time-aligned sequence (red dots). Placeholder frames are assigned negative sequence \#s.

In Fig. 4, the recovered sequence has obviously deviated from the trend of the original sequence at around the $1300^{\text {th }}$ frame (placeholder frames are counted). This phenomenon relates to timeline distortion. In other words, in this example, the timeline is expanded, or tickets are over-consumed. One way to recover the timeline is to use the correction factor calculated from the Ticket Count. Another method is to perform a linear regression on the delay relative to the expected sequence \#, as illustrated in Fig. 5.

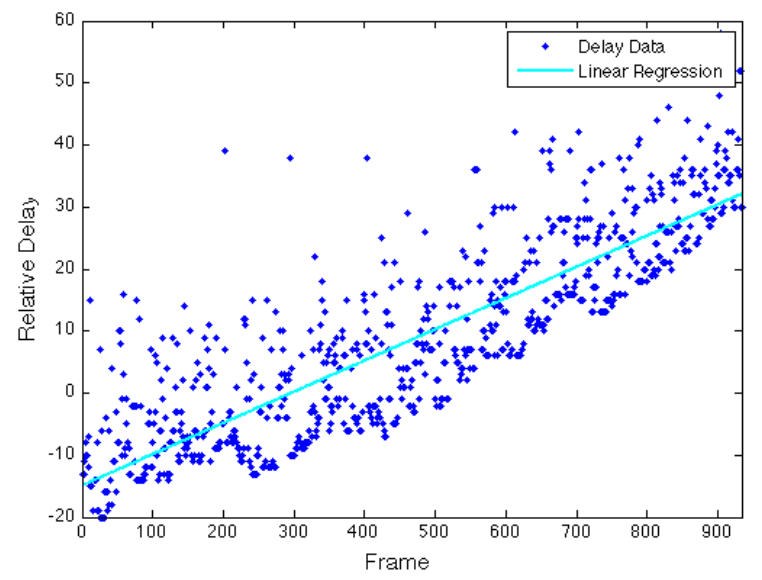

Fig. 5. Linear regression - relative delay to recover the distorted timeline.

A reference frame has the expected sequence \#; it could be a placeholder frame. The expected sequence \# increases by 1 for each time step, returning to 0 after reaching the maximum. This approach estimates the relative delay of every frame, especially the reference frame, which is used to adjust the synchronization of the frame streams from different sensor nodes in a statistical manner.

\section{Pulse Wave Velocity Estimation}

\section{A. Decomposition of the Sensor Data Streams}

The PWV application involves two sensor nodes placed at the wrist (node 1) and the corresponding fingertip (node 2). PWVs are calculated from the time differences between these two PPGs. The first step is to separate the two sensor data streams based on their IDs. Fig. 6 plots two example waveforms in their received order; the data points with negative values represent placeholder frames.

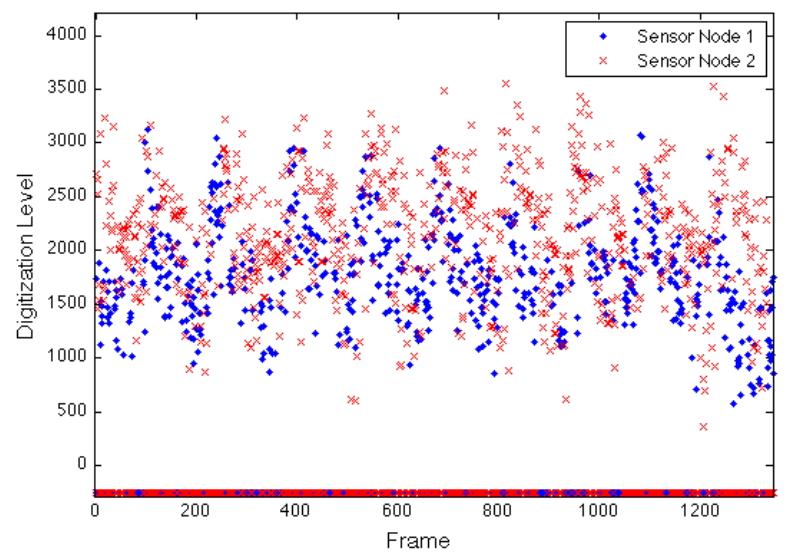

Fig. 6. The original frame sequence decomposed into two PPGs. Each frame carries two AC values, but only one is plotted here.

\section{B. Timeline-Recovered Waveforms}

Sequence \#s are utilized to time-align data points for each sensor node. The rearranged results are depicted in Fig. 7. This time, data points with negative values are from placeholders corresponding to lost frames. Synchronization will be addressed in Section IV.D.

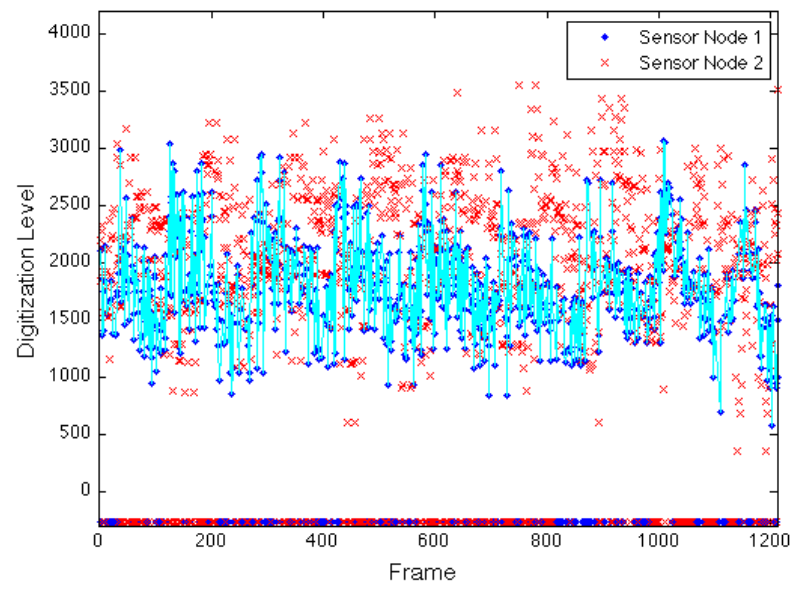

Fig. 7. Two PPGs with data points time-aligned. 


\section{Defragmentation and Curve Fitting}

Although the PPG data points for node 1 (blue dots) are connected with lines in Fig. 7, they are actually fragmented or composed of small data segments. I.e., the sequence \# is not continuous, which is also indicated by the points with negative values. To eliminate the discontinuities, a peak detection algorithm is employed to identify individual PPG cycles, and these cycles are then 'stacked' together; cycles are aligned at the systolic peak position. Fig. 8 shows a representative PPG (blue circles, including at least one whole cycle) averaged from eight cycles worth of discrete, discontinuous data. Note that the $x$-axis is changed to Count, since each frame contains two data points. The sampling frequency $=480 \mathrm{~Hz}$. A $10^{\text {th }}$-order polynomial curve was fitted to these data to estimate the continuous PPG.

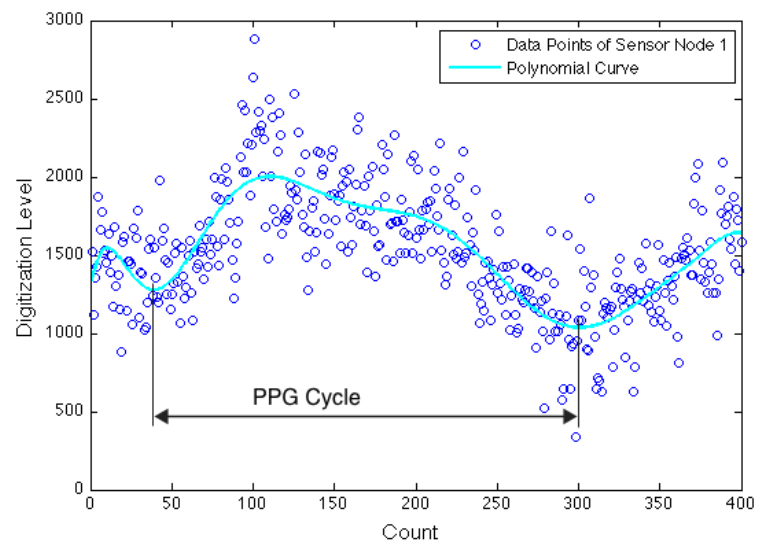

Fig. 8. Representative waveform for eight PPG cycles worth of discontinuous, discrete data.

\section{Synchronization and Feature Extraction}

After the polynomial curves for both nodes are calculated, they need to be placed appropriately, or synchronized, relative to one another, in order to complete the PWV calculation. The positioning process depends on two factors related to both nodes: 1) the corresponding peak position (the first peak of the eight cycles) in the time aligned sequence and 2) the delay relative to the expected sequence \# based on the results of the linear regression method in Section III.E. Fig. 9 shows the relative position of two polynomial curves after statistical synchronization.

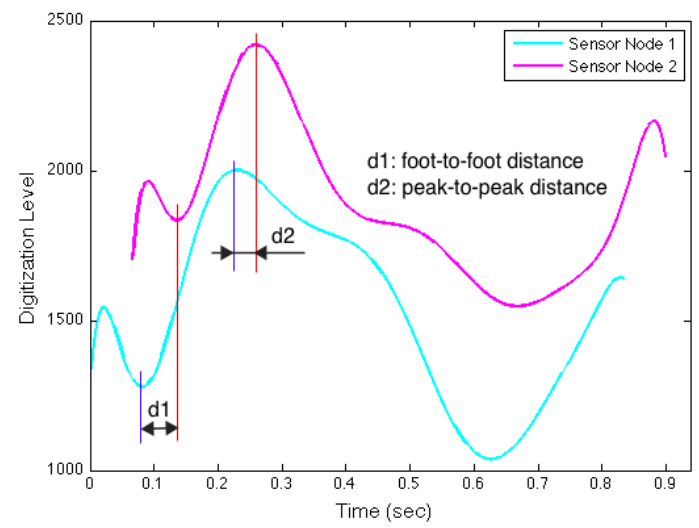

Fig. 9. Statistically synchronized, continuous PPGs from two sensor nodes.
To calculate PWV, corresponding features on the rising slope of the two waveforms could be identified toward the use of the time difference extraction approaches used in [7], which involve taking derivatives of the PPGs. For example, the foot-to-foot and peak-to-peak differences are determined by first finding the roots of the first derivatives and then performing a subtraction (see Fig. 9). In this example, three PWV values are estimated as $5.12 \mathrm{~m} / \mathrm{s}$ at the foot, $5.51 \mathrm{~m} / \mathrm{s}$ at the inflection point, and $9.47 \mathrm{~m} / \mathrm{s}$ at the peak.

\section{CONCLUSION}

This paper presented an application layer scheme for WBANs with sensor nodes that compete for high-speed, real-time communication with the receiver. The lost and wandering frame issue was addressed by assigning a sequence \# to each frame and a time-alignment process. The timeline distortion phenomenon was addressed by a ticket generation and consumption mechanism. A linear regression method provided the relative delay when rearranging the frames that provided information for the statistical synchronization of each sensor.

As a demonstrative application for such a WBAN, PWVs were successfully extracted from the received frames. In other research areas such as digital volume pulse (DVP) analysis [9], the estimated PPGs would help to improve precision, especially in a WBAN scenario.

\section{REFERENCES}

[1] S. Rhee, B. H. Yang, and H. Asada, "Artifact-Resistant, PowerEfficient Design of Finger-Ring Plethysmographic Sensors," IEEE Transactions on Biomedical Engineering, vol. 48, pp. 795-805, July 2001

[2] R. G. Haahr, S. B. Duun, M. H. Toft, B. Belhage, J. Larsen, K Birkelund, and E. V. Thomsen, "An Electronic Patch for Wearable Health Monitoring by Reflectance Pulse Oximetry," IEEE Transactions on Biomedical Circuits and Systems, vol. 6, pp. 45-53, 2012.

[3] M. Tavakoli, L. Turicchia, and R. Sarpeshkar, "An Ultra-Low-Power Pulse Oximeter Implemented With an Energy-Efficient Transimpedance Amplifier," IEEE Transactions on Biomedical Circuits and Systems, vol. 4, pp. 27-38, 2010.

[4] T. O'Donovan, J. O'Donoghue, C. Sreenan, D. Sammon, P. O'Reilly, and K. A. O'Connor, "A Context Aware Wireless Body Area Network (BAN)," 3rd International Conference on Pervasive Computing Technologies for Healthcare, London, April 1-3, 2009, pp. 1-8.

[5] "IEEE 802.15 WPAN Task Group 6 Body Area Networks," 2012, http://www.ieee802.org/15/pub/TG6.html.

[6] M. Chen, S. Gonzalez, A. Vasilakos, H. Cao, and V. C. M. Leung, "Body Area Networks: A Survey," Mobile Networks and Applications, vol. 16, pp. 171-193, 2010.

[7] K. Li and S. Warren, "Initial Study on Pulse Wave Velocity Acquired from One Hand Using Two Synchronized Wireless Reflectance Pulse Oximeters," 33rd Annual International Conference of the IEEE EMBS, Boston, MA, Aug. 30-Sept. 3, 2011, pp. 6907-6910.

[8] K. Li and S. Warren, "A Wireless Reflectance Pulse Oximeter with Digital Baseline Control for Unfiltered Photoplethysmograms," IEEE Transactions on Biomedical Circuits and Systems, vol. 6, pp. 269-278, June 2012.

[9] S. R. Alty, N. Angarita-Jaimes, S. C. Millasseau, and P. J. Chowienczyk, "Predicting Arterial Stiffness From the Digital Volume Pulse Waveform," IEEE Transactions on Biomedical Engineering, vol. 54, pp. 2268-2275, Dec. 2007 